\title{
A Process Meta Model to Support Policy Based Management in Workflow System
}

\author{
Song Ouyang and $\mathrm{Hao} \mathrm{Xu}$ \\ School of Information Science and Engineering, Central South University, Changsha, \\ Hunan, P.R. China 410083 \\ ouyangsong@yahoo.com, \\ xuhao916@gmail.com
}

\begin{abstract}
Policy-based management and process-based management are two main management paradigms that are widely used in information management systems. The foundation of traditional WfMS is process-based management. In this paper we present a process meta model to support policy based management in WfMS. With the new meta model the system has better flexibility in workflow design time and higher adaptability at run-time. It can also keep the business rules consistent across the whole enterprise and provide a uniform method to deal with business rules, resources and security in workflow system.
\end{abstract}

Keywords: Workflow, WfMS.

\section{Introduction}

With the increase of complexity, traditional workflow management systems (WfMS) are facing many challenges in meeting the requirements of flexibility and adaptability and implementing resource management and security control [2]. To improve flexibility and adaptability, a Petri net formalism is presented to analyze structural change in the workflow process modeling [3]. A new workflow modeling method is proposed to improve flexibility and adaptability of system [4]. A meta-model is proposed in [5] to support dynamic changes of workflow process and enable the dynamic characteristics. Similar approach can be found in [6]. To address various software and/or hardware resources management issues, a resource management system that can handle a large number of workflow resources is proposed [7].

The foundation of a traditional WfMS is process-based management. The features of this management method are: the execution of each step of a task is based on an instruction and its parameters; the description of the instruction is imperative and it invokes execution components directly; after one instruction completed, another predefined instruction is initiated based on the pre-defined rules and conditions. The controlling mechanism between steps is accurate. In some workflow systems, better flexibility and adaptability in flow control are achieved through expanding the types of activities and adding more collaboration between activities. For the applications with comprehensive requirements in resources management and security control, 
however, it is difficult to improve the flexibility and adaptability by using similar methods that are used in control flow since the requirements are more complicated.

Another management paradigm widely used in information management systems is policy-based management. The features of this management method are: the execution of each step of a task is based on the objective or behavior constraints; the description of instruction is declarative and it can not invoke execution components directly in most cases; after one step completed the subsequence step will be executed based on the analysis of active policies and the state of whole system.

Although some workflow systems used policies to deal with resources and security control, it is not real policy-based management system. The distinct features of policy-based management from process-based management are: declarative instruction, a centralized policy repository, dynamic policy transformations and looking up for policies, independent policy engine, and decision-making based on policies.

To meet the challenges we proposed to integrate the policy-based management in a WfMS [1]. This paper presents a process meta model to support policy based management in a WfMS. With the new meta model the system has better flexibility in workflow design time and higher adaptability at run-time.

\section{Process Meta-model to Support Policy Based Management}

Workflow process meta-model is the base for process definition modeling. A new process meta-model to support policy management is shown in Fig.1.

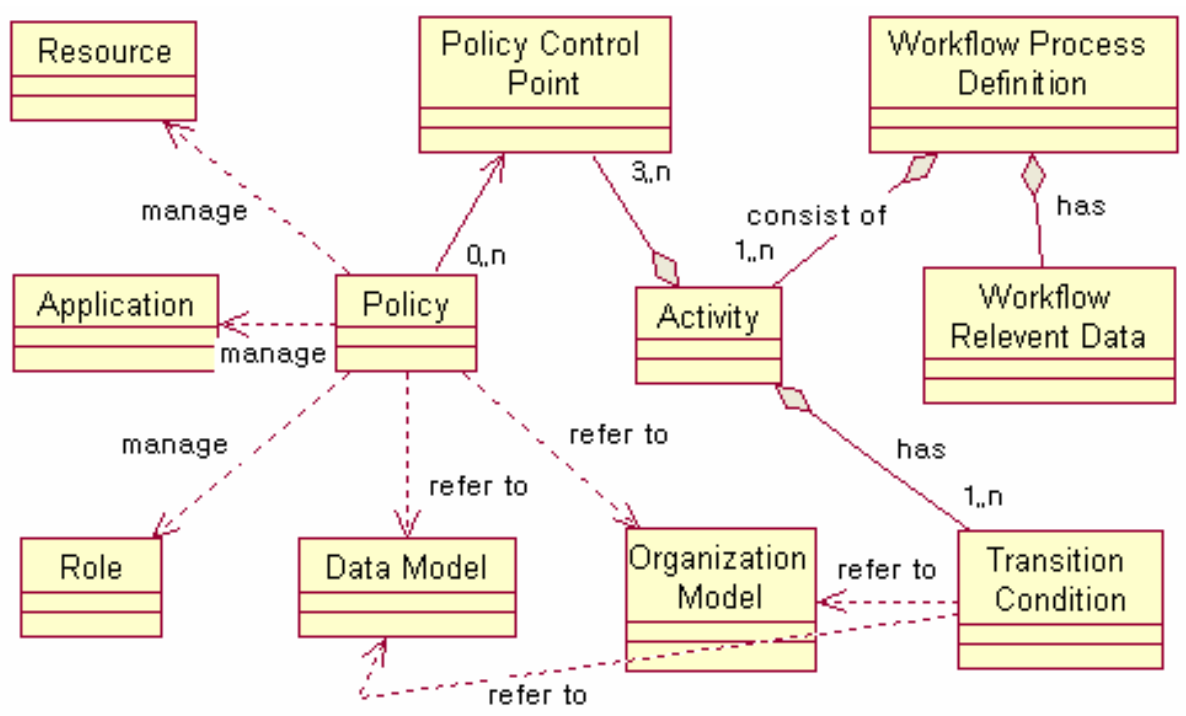

Fig. 1. A process meta-model to support policy based management 
The main elements in the model are described as follows.

Process Activity: A logical step or description of a piece of work that contributes toward the achievement of a process.

Workflow Relevant Data: Data that is used by a WfMS to determine the state transition of a workflow instance.

Transition Condition: Criteria for moving, or state transitioning, from the current activity to the next activity(s) in a process instance.

Organization Model: The model of an enterprise's organizational structures.

Resource/Application/Role: They are managed through late-bound components (Subjects) to perform the specific behavior described by a policy.

Policy Control Point: The proper point defined in workflow to apply policies.

The new meta model has some new features:

(1) It provides more means to develop a process definition in workflow system. Using policies the complicated business rules, the local activity goals, and the constraints for resources can be described in a straight way. The flexibility in workflow design time is much better than that in a traditional process-based system.

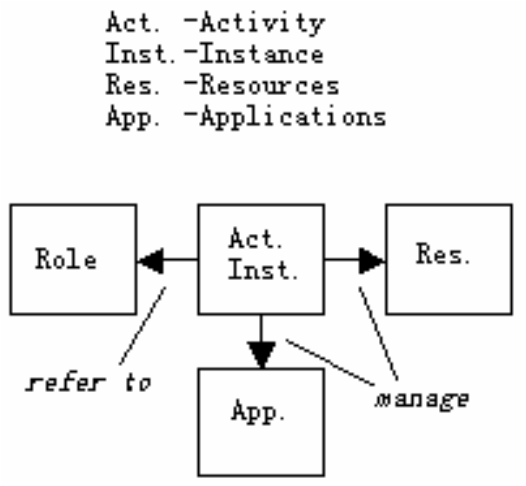

(a)

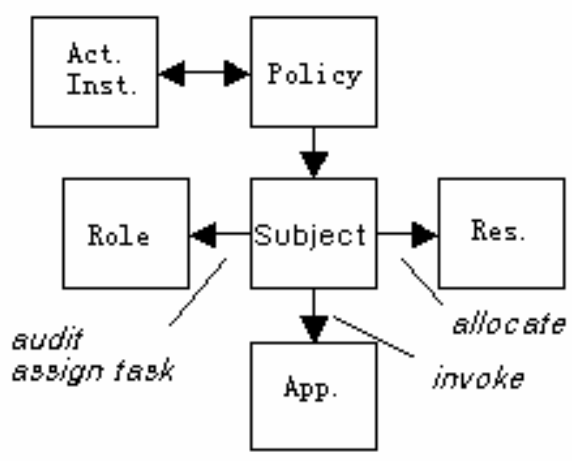

(b)

Fig. 2. Relations of activities, roles/participant, resources, and applications

(2) It makes it possible to separate the activities of process from the operations on roles, resources, and the applications. In the traditional process-based system, the relations between activities, roles, resources, and applications are shown in Fig.2 (a). It can be seen that workflow engine needs to deal with them directly. It results in some difficult in system design and system maintenance. Fig. 2(b) shows the new relations. All the operations on resources, roles and the applications are done by policy engine through the late-bound components. The separation benefits the system design and the enterprise administration. 


\section{Advantages of New Approach}

Integrate the policy-based management in traditional WfMS has many advantages:

Firstly, it improves flexibility and adaptability. For example, a loan application usually has many business rules to deal with. This can be done by a traditional process based workflow system. For some business rules such as determine whether the supplied information is complete, credit scoring and other risk assessment, execute the required level of approval and ensure regulatory compliance it is better using policy-based management. With policy-based management, business managers can specify only the policies or local objectives for these business rules. Policies can also describe much complex requirements of resources and security control. So it has better flexibility in design time. At the run-time the active policies in policy repository can be changed dynamically, a policy and the components to execute this policy can be late bound to achieve higher adaptability.

Secondly, a centralized policy repository and an independent policy engine to execute policies make it possible to keep the business rules consistent across the whole enterprise.

Lastly, it is possible to use a uniform method to deal with resources and security controls and business rules. This benefits the system design.

\section{Conclusion and Future Work}

The new process meta model described in this paper defines new elements to support policy-based management in WfMS. Based on the model a workflow system has better flexibility and adaptability. We have some experience on developing the policy engine in network management systems. The future work is to make expansions to the traditional workflow engine to achieve close collaboration with the policy engine.

\section{References}

1. Song Ouyang, "Integrate Policy based Management and Process based Management-A New Approach for Workflow Management System", IEEE proceedings of CSCWD2006, Nanjing, China (2006).

2. Shi MeiLin, Yang GuangXin, Xiang Yong, Wu ShangGuang, "Workflow Management Systems: A Survey", Proceedings of IEEE Intl Conf on Communication Technology, Beijing (1998)

3. CA Ellis, K. Keddara, G. Rozenberg., "Dynamic change within workflow systems", Proceedings of International, ACM Conference, COOCS '95, Milpitas, CA, August. (1995)

4. Fan YuShun, Wu Cheng, "Research on a Workflow Modeling Method to Improve System Flexibility", Journal of Software, Vol. 13 (2002)

5. Sun ReiZhi, Shi MeiLin, "A Process Meta-Model Supporting Dynamic Change of Workflow”, Journal of Software, Vol. 14 (2003)

6. Zhao Wen, Hu WenHui, Zhang ShiKun, Wang LiFu, "Study and Application of a Workflow Meta-Model", Journal of Software, Vol. 13 (2003)

7. Weimin Du, Ming-Chien Shan: "Enterprise Workflow Resource Management". RIDE (1999) 